VOLUMEN 37 | N 1 | ABRIL 2019

FECHA DE RECEPCIÓN: 12/02/2019 FECHA DE APROBACIÓN: 17/04/2019 FECHA PUBLICACIÓN: $\quad 30 / 04 / 2019$

1. Universidad San Francisco de Quito

2. Hospital Juárez de México

\begin{tabular}{l|l} 
Caso & Clinical \\
Clínico & Case
\end{tabular}

Correspondencia:

mari_fer19851ahotmail.com

Dirección:

Luis Cordero y Juan Jaramillo 5-86

Código Postal:

010107

Teléfono:

0979128896

Cuenca - Ecuador

\section{Manejo endovascular de aneurisma de la vena de Galeno: reporte de un caso}

\author{
Endovascular management aneurysm of Galen vein: a case \\ report \\ Guillermo Quinde María Fernanda', Moctezuma Ramírez Luis²
}

\section{RESUMEN}

La malformación aneurismática de la vena de Galeno (MAVG) es la fístula arteriovenosa menos frecuente dentro de la clasificación de las malformaciones arteriovenosas intracraneales, de alta mortalidad que las técnicas endovasculares disminuyen. Se presente el caso de un niño de 2 años 4 meses sometido a tratamiento endovascular por tercera ocasión, quien fue diagnosticado desde la etapa prenatal de MAVG, se programó y ejecutó embolización endovascular en vasos de alimentación que tenían $60 \%$ de obliteración, luego del cual permanece estable en la unidad de cuidados intensivos y posteriormente es dado de alta sin complicaciones.

Palabras Clave: malformaciones de la vena de Galeno, procedimientos endovasculares, malformaciones arteriovenosas intracraneales, niño.

\section{ABSTRACT}

The aneurysmal malformation of the vein of Galen (VOGM) is the arteriovenous fistula less frequent within the classification of intracranial arteriovenous malformations, with high mortality that endovascular techniques decrease. A case of a 2-years and 4-months-old boy who were submitted to an endovascular treatment for the third time is presented. The patient was diagnosed from the prenatal stage of VOGM, an endovascular embolization was programmed and executed in feeding vessels with $60 \%$ of obliterated, after that the patient stays stable in the intensive care unit and finally the patient is discharged without complications.

Keywords: vein of Galen malformation, endovascular procedures, intracranial arteriovenous malformations, child. 


\section{INTRODUCCIÓN}

Las malformaciones arteriovenosas intracraneales en niños representan el $30 \%$ de todas las malformaciones vasculares, siendo la malformación aneurismática de la vena de Galeno (MAVG) el 1\% de todas las intracraneales, fue descrita por Steinhel en 1895 [1]. El manejo interdisciplinario para su tratamiento se restringía a grandes centros pediátricos $[2,3]$.

Hasta la llegada de los tratamientos endovasculares a fines de la década de 1980, la mortalidad con el tratamiento quirúrgico abierto de estas lesiones es alta (37.4\%). Los neonatos tuvieron el peor pronóstico con una mortalidad superior al 90\% [4]. Posteriormente, el desarrollo de las técnicas endovasculares han ofrecido una ventaja sustancial, es así como investigaciones en el año 2006 reportan una tasa de mortalidad del $10.6 \%$, con el $74 \%$ de los que sobrevivieron sin una morbilidad neurológica significativa [5].

Durante la infancia, el líquido cefalorraquídeo se reabsorbe a través del epéndimo ventricular y parénquima cerebral en las venas medulares. La presencia MAVG puede elevar la presión del seno venoso, que se transmite a su vez a la cortical y finalmente las venas medulares; esto dará lugar a edema del parénquima cerebral y deterioro de la oxigenación que conduce a la atrofia subependimaria y en casos severos un "síndrome fusión cerebral" progresiva $[6,7]$. La presentación más común de MAVG resulta del tamaño de la propia derivación, imponiendo precarga elevada en el lado derecho del corazón que conduce a la insuficiencia cardiaca que puede progresar a insuficiencia multiorgánica [8].

La MAVG representa probablemente una fístula arteriovenosa (FAV) en la pared de un canal vascular persistente embriológicamente, conocido como la vena prosencefálica media. Si las venas prosencefálicas medias no regresan normalmente, puede persistir una conexión fistulosa con las arterias primitivas coroideas, resultando así en esta malformación [2].

La terapia endovascular es una modalidad de tratamiento seguro y eficaz para este tipo de lesiones, el beneficio y objetivo de la embolización es regular la vascularización, permitiendo el desarrollo normal de un niño sin déficit neurológico [9].

\section{PRESENTACION DEL CASO}

Paciente de 2 años 4 meses, masculino, originario y residente de México, con antecedentes heredofamiliares de carga genética para diabetes II. Nacido a las 39.5 semanas de gestación vía cesárea por doble circular de cordón; con controles prenatales completos, diagnóstico prenatal de probable alteración del polígono de Willis, al nacimiento presentó un peso de $3.5 \mathrm{~kg}$, talla de $49 \mathrm{~cm}$. Ingreso a UCIN (unidad de cuidados intensivos neonatales) durante dos meses por dificultad respiratoria y sepsis neonatal. Diagnosticado de septum inter atrium con foramen oval de $2 \mathrm{~mm}$ durante los primeros meses de edad, además de insuficiencia tricúspidea importante, dilatación de cavidades derechas con hipertensión pulmonar, es tratado con sildenafilo $2 \mathrm{mg} \mathrm{QD}$, espirinolactona $7 \mathrm{mg} \mathrm{QD}$, furosemida $7 \mathrm{mg}$ QD y $3.5 \mathrm{mg}$ hidroclorotiazida por 2 meses.

Actualmente se descarta foramen intraauricular por ecocardiografia de control previo al segundo procedimiento.

Antecedentes personales: el paciente registra dos embolizaciones previas, embolización parcial en un $10 \%$ con 1 dispositivo tipo coil y ónix (que es un medio líquido no adhesivo); $y$, la segunda embolización en un 20 \% con ónix.

Padecimiento actual: paciente estable, ingresa de forma programada a cargo del servicio de neurocirugía para realización de embolización de MAVG.

Examen físico: signos vitales: presión arterial: 120/80 mmHg; frecuencia cardiaca: 116/minuto; frecuencia respiratoria: 20/minuto; temperatura. $36.5^{\circ} \mathrm{C}$. Peso: $10.7 \mathrm{~kg}$, talla: $86 \mathrm{~cm}$, IMC $14.5 \mathrm{~kg} /$ m2. Cabeza: Braquicéfalo. Precordio: frecuencia de 116/minuto sin arritmias. Abdomen: cicatriz en relación a cirugías anteriores. Exploración neurológica: Glasgow 15/15. Retraso psicomotor a expensas de desarrollo de destrezas motoras (caminar). Nervios craneanos: sin alteración. Reflejos musculocutáneos: abdominal superior T8-T9 y abdominal inferior T11- T12 presentes, no hay evidencia de signos patológicos de Babinski ni Hoffman. Reflejos musculares: reflejo mandibular $(++)$, bicipital C5 (++) derecho e izquierdo, tricipital C7 (++) derecho e izquierdo $(++)$, flexor de los dedos $\mathrm{C} 7(++)$ derecho e izquierdo (++), patelar L4 (++) derecho e izquierdo (++), Aquileo S1 (++) derecho e izquierdo 
$(++)$, normotonía en las cuatro extremidades. Sensibilidad al dolor presente. Cerebelo: no valorable. Meníngeos: sin datos de irritación. Marcha: no valorable.

Laboratorio: leucocitos: 8,420 , Neutrófilos: 28.5 \%, Linfocitos: $56.4 \%$, Plaquetas: 265,000 . TP: 14.6 seg. TPT 25.2 seg. Creatinina $0.31 \mathrm{mg} / \mathrm{dl}$. Glucosa: $65 \mathrm{mg} / \mathrm{dl}$ Urea: $11 \mathrm{mg} / \mathrm{dl}$. Cloro: $102 \mathrm{mEq} / \mathrm{K}$ : $4.4 \mathrm{mEq} / \mathrm{l}$.

Imágenes: en las Imágenes $\mathrm{N}^{\circ} 1, \mathrm{~N}^{\circ} 2$ y $\mathrm{N}^{\circ} 3$, se muestra el estado prequirúrgico con resonancia magnética y angioresonancia, observándose la condición de la malformación aneurismática.

\section{Imagen $\mathbf{N}^{\circ} 1$}

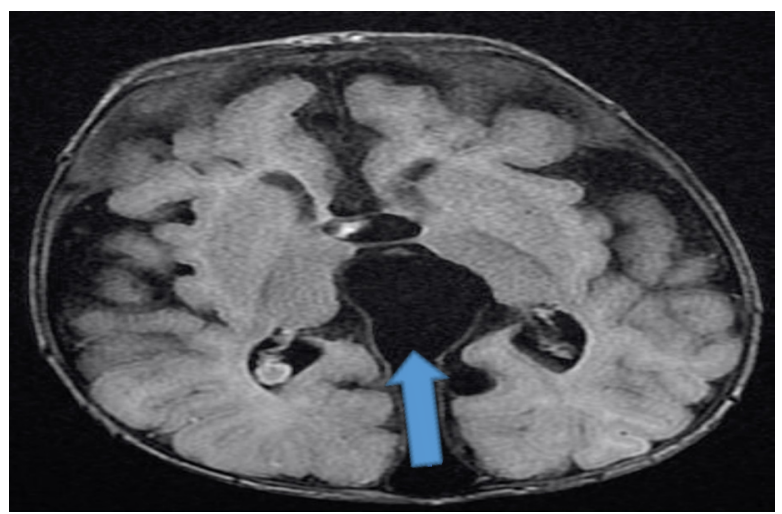

Resonancia magnética ponderación T1, corte axial, se identifica vacio de señal interventricular, interhemisférico que conecta con el seno longitudinal superior.

\section{Imagen $\mathrm{N}^{\circ} 2$}

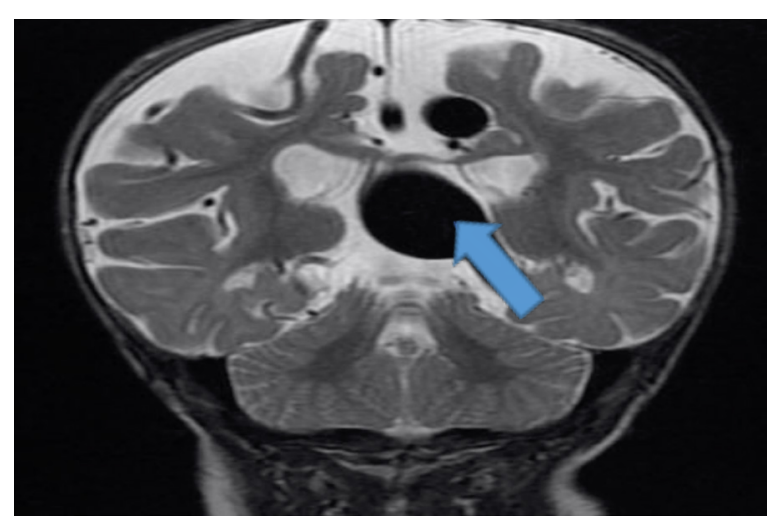

Resonancia magnética, corte coronal ponderación T2, vacío de señal, interhemisferico, intertalámico, subcalloso y supra-cingular; en relación con colector vascular anómalo.

\section{Imagen $\mathbf{N}^{\circ} 3$}

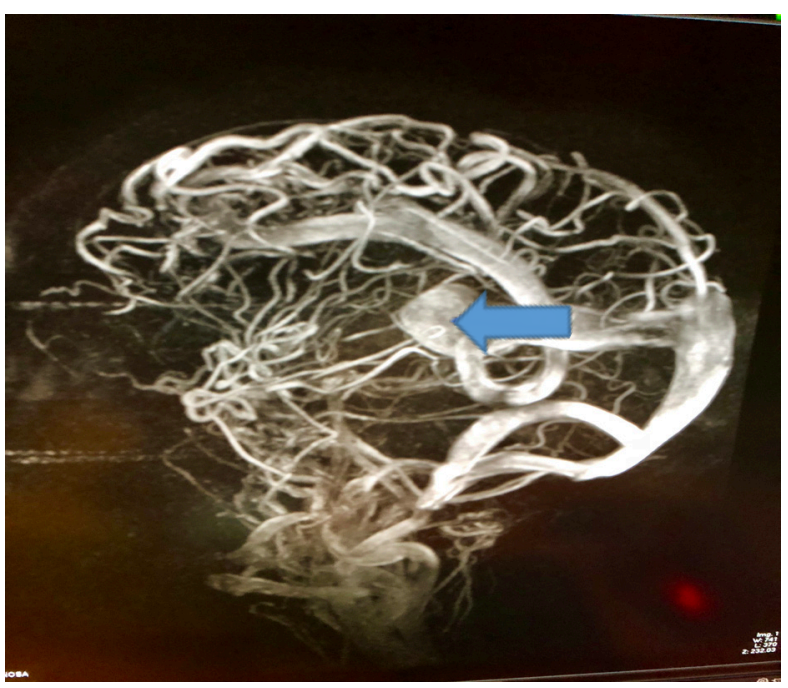

Angioresonancia, ponderacion en fase venosa se identifica vena prosencefálica de Markowsky.

Diagnóstico topográfico: Sistema venoso cerebral. Diagnóstico etiológico: Vascular.

Diagnóstico nosológico: Pseudoaneurismática de la vena de galeno parcialmente embolizado. Pronóstico: Reservado a evolución. Plan: Angiografía y embolización de lesión

\section{Tratamiento}

Después de confirmar el consentimiento informado y la inanición adecuada, se colocan todos los monitores estándar: electrocardiograma, capnógrafo ETCO $_{2}$ y oxímetro de pulso, se adjunta sonda manguito de presión arterial. Intra-operativamente el ritmo cardíaco se mantuvo estable, la saturación era $96 \%, \mathrm{ETCO}_{2}$ a $30 \mathrm{mmHg}$. El niño fue inducido con sevoflurano usando circuito semicerrado pediatrico y después de confirmar la ventilación, se administro relajante muscular vencuronio $1 \mathrm{mg} \mathrm{y}$ el paciente fue intubado con un tubo endotraqueal $4.0 \mathrm{~mm}$ sin manguito. La anestesia se mantuvo con sevoflurano, aire: oxígeno y fentanilo. Se canuló la arteria femoral izquierda. El paciente se presenta normotenso durante todo el procedimiento. Se 
logró embolizar los vasos de alimentación que tenían $60 \%$ de obliteración. Una hora después del acto operatorio es conducido a la unidad de cuidados intensivos con los siguientes signos vitales: presión arterial: 108/65; frecuencia respiratoria: 25/ minuto; saturación de O2: 93\%; frecuencia cardiaca: 103/minuto; RAMSAY: 2; EVA: 0. En las imágenes $\mathrm{N}^{\circ} 4$ y $\mathrm{N}^{\circ} 5$ se observan los resultados del procedimiento.

\section{Imagen $N^{\circ} 4$}

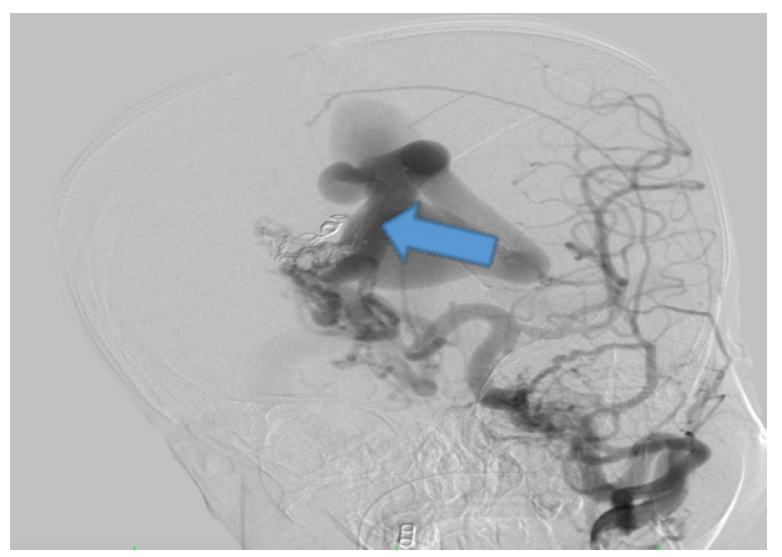

Vista angiográfica de la malformación de la vena de Galeno

\section{Imagen $\mathbf{N}^{\circ} 5$}

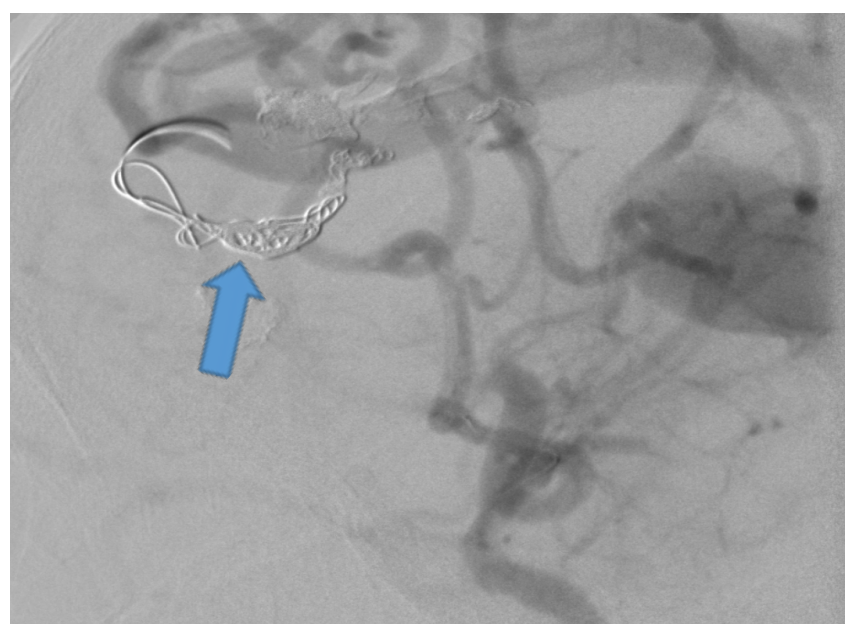

Disminución del flujo en la alimentación de recipiente después de la embolización

La estancia en cuidados intensivos no registra complicaciones, el paciente es llevado al servicio de Pediatría estable para el control posoperatorio y fue dado de alta en mejores condiciones.
El menor acude a controles periódicos por consulta externa de Neurocirugía y Pediatría, persistiendo retraso psicomotriz para la edad y aún está por determinar su pronóstico a largo plazo.

\section{DISCUSIÓN}

En las etapas tempranas del desarrollo fetal no existen per se arterias, venas y capilares. Inicialmente, todos los vasos cerebrales embrionarios son simplemente tubos endoteliales. Cuando se desarrolla la función cardíaca y comienza el flujo sanguíneo intracraneal, algunos de estos canales primitivos vasculares normalmente evolucionan hacia vasos aferentes (arteriales), mientras que otros se convierten en eferentes o venosos. La persistencia de estas conexiones primordiales directas entre la futura circulación arterial y venosa, combinada con agenesia o pobre desarrollo de la red capilar puede ser el origen de las Malformaciones Vasculares Cerebrales [10].

En la actualidad se han reclasificado de acuerdo a una combinación de factores anatómicos e histopatológicos, a la presentación clínica, al comportamiento biológico y a las características de imagen; la MAVG está incluida dentro de las fístulas arteriovenosas (FAV) menos habituales. Las FAV cerebrales (FAVc) se caracterizan por existir comunicación directa entre arterias piales y canales venosos. Hay dos formas principales: a) la malformación aneurismática de la vena de Galeno (MAVG), que aparece en neonatos y lactantes y b) la FAV pial o subependimaria [2].

Las MAVG son anomalías raras congénitas (menos de 1/25.000) de la circulación intracraneal que constituyen el $1 \%$ de todas las malformaciones vasculares intracraneales $[2,3]$. El origen de MAVG es de una vena media embrionaria persistente del prosencéfalo. En el útero, insuficiencia cardiaca secundaria a MAVG es raro porque la circulación placentaria proporciona una circulación de baja resistencia [2].

Una opción para la evaluación del recién nacido con MAVG se basa en la puntuación de Bicktre para determinar las posibles opciones de tratamiento. Esta escala de 21 puntos proporciona puntos para la gravedad de los signos y síntomas relacionados con los sistemas cardíaco, pulmonar, neurológico, hepático y renal (Tabla N¹). Los valores clínicos y de laboratorio se utilizan para calcular 
la puntuación de Bicêtre para el recién nacido que se presenta con VGAM. Una puntuación de $<8$ de 21 sugiere un pronóstico casi fatal, y el infante se considera demasiado inestable para la embolización de emergencia. Una puntuación entre 8 y 12 caracteriza a los recién nacidos que tienen más probabilidades de beneficiarse de la embolización emergente. Un puntaje $>12$ sugiere que los bebés que son candidatos para el tratamiento médico de su insuficiencia cardiopulmonar. El tratamiento médico continúa hasta los 5 meses de edad, cuando su tamaño mayor disminuye los riesgos de embolización prolongada [11].

\section{Tabla No 1}

Escala de evaluación neonatal de Bicetre

\begin{tabular}{|c|c|c|c|c|c|}
\hline Points & Cardiac funcion & Cerebral function & Respiratory function & Hepatic function & Renal function \\
\hline 5 & Normal & Normal & Normal & ---------- & ------------ \\
\hline 4 & $\begin{array}{l}\text { Overload, no } \\
\text { medical treat- } \\
\text { ment }\end{array}$ & $\begin{array}{l}\text { Subclinical, isolated } \\
\text { EEG abnormalities }\end{array}$ & $\begin{array}{l}\text { Tachypnea, finishes } \\
\text { bottle }\end{array}$ & ---------- & ----------- \\
\hline 3 & $\begin{array}{l}\text { Failure; stable } \\
\text { with medical } \\
\text { treatment }\end{array}$ & $\begin{array}{l}\text { Nonconvulsive } \\
\text { intermittent neuro- } \\
\text { logic signs }\end{array}$ & $\begin{array}{l}\text { Tachypnea, does not } \\
\text { finish bottle }\end{array}$ & $\begin{array}{l}\text { No hepatomegaly, } \\
\text { normal hepatic func- } \\
\text { tion }\end{array}$ & Normal \\
\hline 2 & $\begin{array}{l}\text { Failure; not sta- } \\
\text { ble with medical } \\
\text { treatment }\end{array}$ & Isolated convulsion & $\begin{array}{l}\text { Assisted ventilation, } \\
\text { normal saturation } \\
\text { FiO2 menor } 25 \%\end{array}$ & $\begin{array}{l}\text { Hepatomegaly, nor- } \\
\text { mal hepatic function. }\end{array}$ & Transient anuria \\
\hline 1 & $\begin{array}{l}\text { Ventilation nec- } \\
\text { essary }\end{array}$ & Seizures & $\begin{array}{l}\text { Assisted ventilation, } \\
\text { normal saturation } \\
\text { FiO2 mayor } 25 \%\end{array}$ & $\begin{array}{l}\text { Moderate or tran- } \\
\text { sient hepatic insuffi- } \\
\text { ciency. }\end{array}$ & $\begin{array}{l}\text { Unstable diuresis } \\
\text { with treatment }\end{array}$ \\
\hline 0 & $\begin{array}{l}\text { Resistant to } \\
\text { medical therapy }\end{array}$ & Permanent signs & $\begin{array}{l}\text { Assisted ventilation, } \\
\text { desaturation }\end{array}$ & $\begin{array}{l}\text { Abnormal coagula- } \\
\text { tion, elevated en- } \\
\text { zymes }\end{array}$ & Anuria. \\
\hline
\end{tabular}

Score <8: no intervention, 8-12: immediate/emergency embolization, $>12$ : delayed treatment over the age of 5 months.

Fuente: Vein of Galen Aneurysmal Malformations: An Ultrasonographic Incidental Finding—A Case Report.

El caso clínico presentado corresponde una Escala de Bicetre mayor a 12, razón por la cual se inició la intervención endovascular a partir de los seis meses de edad y culmino a los 2 años 4 meses con la tercera embolización; la respuesta clínica es favorable y los controles posteriores por el servicio de Neurología determinarán la evolución en el retraso psicomotriz.

La insuficiencia cardiaca se produce como el gran volumen de flujo de la derivación MAVG se descarga en el atrio derecho y la circulación pulmonar, con la vasoconstricción pulmonar y la hi- pertensión pulmonar resultante en la insuficiencia del ventrículo derecho [3].

Con la pérdida de la placenta al nacer, hasta el $70 \%$ del gasto cardíaco se dirige a la derivación arteriovenosa de baja resistencia de MAVG que permite el retorno directo del volumen de flujo grande para el lado derecho del corazón [2]. En una serie publicada por $\mathrm{Li} \mathrm{AH}$., y colaboradores en el 2011 , el $46 \%$ de los pacientes presentó MAVG con insuficiencia cardiaca de alta salida [9]. 
Por lo tanto, el diagnóstico de una malformación arteriovenosa requiere una colaboración interdisciplinaria intensiva de obstetras, pediatras, neurorradiólogos y neurocirujanos. Si se presenta la insuficiencia cardíaca prenatal y/o daños cerebrales, el pronóstico para el paciente es malo [1].

Los recientes avances en el manejo de estos pacientes, en particular el uso de técnicas endovasculares en un entorno dedicado a cuidados intensivos neonatales, han alterado significativamente este pronóstico sombrío [7].

La intervención neurorradiológica con embolia transcatéter es el procedimiento de elección para ocluir el MAVG debido al hecho de que es menos invasiva y tiene una tasa de supervivencia más alta que los procedimientos neuroquirúrgicos abiertas $[1,12]$.

La embolización de las arterias de alimentación y de las venas drenantes puede provocar una disminución del flujo sanguíneo y una mayor tasa de supervivencia en los niños con MAVG [13].

Berenstein A., y colaboradores realizaron un análisis retrospectivo de 45 pacientes con MAVG tratados entre enero de 2004 y abril de 2015. Después de las exclusiones, se incluyeron 45 pacientes, encontraron una mortalidad del $4.4 \%$ [13].

El manejo óptimo de los pacientes con un MAVG se logra solo a través del enfoque integral y multidisciplinario que ofrecen los centros de atención terciaria especializados. No es infrecuente que estos pacientes se sometan a una serie de procedimientos de tratamiento endovascular en la suite neurorradiológica. El grado de apoyo ventilatorio y hemodinámico puede cambiar rápidamente, y los anestesiólogos deben estar preparados para valorar los objetivos predefinidos. Ciertamente, la monitorización invasiva y el acceso venoso central son necesarios en estos pacientes, ya que es una forma confiable de administrar grandes cantidades de soporte de volumen intravascular si es necesario. [14].

El manejo de la anestesia y cuidados intensivos de estos pacientes sigue siendo un reto debido al riesgo de complicaciones periprocedimiento como la insuficiencia cardíaca congestiva (CCF) y el infarto cerebral. Por otra parte, la gestión de las vías respiratorias de recién nacidos y lactantes con macrocefalia secundaria a la MAVG e hidrocefalia presenta un desafío único [15-17].

\section{CONCLUSIONES}

Se presenta el caso de un niño de 2 años 4 meses en su tercera terapia de angioembolización que es el tratamiento de elección para este tipo de malformaciones previa valoración clínica utilizando escalas como la Evaluación neonatal de Bicetre, se consigue por vía endovascular recuperar para vasos de alimentación que tenían $60 \%$ de obliteración.

\section{INFORMACIÓN DE LOS AUTORES}

- Guillermo Quinde María Fernanda. Posgradista de Anestesiologia. Universidad San Francisco de Quito.

ORCID: https://orcid.org/0000-0002-7453-0181

- Moctezuma Ramírez Luis. Profesor de Neuroanestesia de la UNAM. Hospital Juárez de Mexico. ORCID: https://orcid.org/0000-0002-5743-9693

\section{CONTRIBUCIÓN DE LOS AUTORES}

GF, ML, Realizaron la revisión bibliográfica, redacción y análisis crítico del manuscrito. Todos los autores leyeron y aprobaron la versión final del artículo.

\section{CONFLICTO DE INTERESES}

Los autores declaran no tener conflicto de intereses en la presente investigación.

\section{FUENTES DE FINANCIAMIENTO}

Ninguna

\section{REFERENCIAS BIBLIOGRÁFICAS}

1. Stephan S, Rodesch G, Elolf E, Wiemann D, Jorch G, Vein of Galen Aneurysmal Malformations: An Ultrasonographic Incidental Finding-A Case Report. Case Reports in Pediatrics. 2012; 1-6 Disponible en: https://www. hindawi.com/journals/cripe/2012/824284/ 
2. Osborn AG. Angiografía Cerebral Diagnostico. Segunda Edición. Washington DC: Editorial Marban; 2017. p 291.

3. Gupta AK, Varma DR. Vena de Galeno malformaciones: Revisión. Neurol la India. 2004; 52: 43-53. Disponible en: https://www.ncbi. nlm.nih.gov/pubmed/7064645.

4. Hansen D, Kan PT, Reddy GD, Mohan AC, Jea A, Lam S. Pediatric knowledge update: Approach to the management of vein of Galen aneurysmal malformations in neonates. Surg Neurol Int. 2016;7(2):S317-21. Disponible en: http://www.surgicalneurologyint.com/surgicalint-articles/pediatric-knowledge-update-approach-to-the-management-of-vein-of-galen-aneurysmal-malformations-in-neonates

5. Johnston IH, Whittle IR, Besser M, Morgan MK. Vein of Galen malformation: diagnosis and management. Neurosurgery. 1987;74758.Disponible en: https://www.ncbi.nlm.nih. gov/pubmed/3601022

6. Gomez DG, Dibenetto AT, Pavese AM, et al. Development of arachnoid villi and granulations in man. Acta Anat (Basel) 1981;11:24758 Disponible en: https://www.ncbi.nlm.nih. gov/pubmed/7064645

7. Gailloud P, et. al. Diagnosis and Management of Vein of Galen Aneurysmal Malformations. Journal of Perinatology. 2005;25(8), 542-551. Disponible en: https://www.nature.com/articles/7211349

8. Chul Suh D, Alvarez $\mathrm{H}$, Bhattacharya JJ, et al. Intracranial haemorrhage within the first two years of life. Acta Neurochir (Wien) 2001;143:997-1004. Disponible en: https:// www.ncbi.nlm.nih.gov/pubmed/17053602

9. Lasajunias PL, Chng SM. The management of vein of Galen aneurysmal malformations. Neurosurgery [Internet]. 2006;59:53-184. Disponible en: https://www.ncbi.nlm.nih.gov/pubmed/17053602
10. Lasjaunias $\mathrm{PL}$, et. al. The management of vein of Galen aneurysmal malformations," Neurosurgery, 2006;59(5) S184-S194, 2006.

11. Stephan S, Vein of Galen Aneurysmal Malformations: An Ultrasonographic Incidental Finding-A Case Report, Case Reports in Pediatrics, 2012: 824284, doi: 10.1155/2012/824284 Disponible en : https://www.hindawi.com/journals/cripe/2012/824284/cta/

12. $\mathrm{Li}, \mathrm{AH}$, Armstrong, $\mathrm{D}$, TerBrugge, KG. Endovascular treatment of vein of Galen aneurysmal malformation: Management strategy and 21-year experience in Toronto. J Neurosurg Pediatr. 2011;7 3-10. Disponible en: https:// thejns.org/pediatrics/abstract/journals/j-neurosurg-pediatr/7/1/article-p3.xml

13. Berenstein A, Paramasivam S, Sorscher M, Molofsky W, Meila D, Ghatan S. Vein of Galen Aneurysmal Malformation: Advances in Management and Endovascular treatment. $\mathrm{Neu}$ rosurgery 2019;84(2):469-478. Disponible en: https://academic.oup.com/neurosurgery/ articleabstract/84/2/469/5026364? redirectedFrom=fulltext

14. G. Beucher, C. Fossey, F. Belloy, B. Richter, M. Herlicoviez, and M. Dreyfus, "Antenatal diagnosis and management of vein of Galen aneurysm: review illustrated by a case report," Journal de Gynecologie Obstetrique et Biologie de la Reproduction, 2005;34(6)613-619.

15. McConnell ME, Aronin P, Vitek JJ. La insuficiencia cardíaca congestiva en los recién nacidos debido a una malformación arteriovenosa intracraneal: El tratamiento endovascular. Pediatr Cardiol 1993;14 : 102-6.

16. Shah-Farhat A, Alizadeh-Kaseb A, Khorakian F, Mohammadzadeh A, Saeidi R- Vein of Galen Aneurysmal Malformation in a Neonate: A Case Report. Iranian Journal of Neonatology. 2016;7(2)45-48. Disponible en: http://ijn. mums.ac.ir/article_7121.html 
17. Puccinelli $F$, et. al. Embolization of cerebral arteriovenous shunts in infants weighing less than $5 \mathrm{~kg}$. J Neurosurg Pediatr. 2019 Feb 22:1-9. doi: 10.3171/2018.11.PEDS1865. [Epub ahead of print]. 